\title{
Schützt Acetylsalicylsäure vor einer erneuten Thromboembolie?
}

Fragestellung: Kann Acetylsalicylsäure Rezidive von venösen Thromboembolien verhindern?

Hintergrund: Gängige Lehrbuchkenntnis ist, dass orale Antikoagulanzien zur Prophylaxe von venösen Thromboembolien wirksam sind und Thrombozytenfunktionshemmer zur Prophylaxe von arterio-arteriellen Embolien. Ob Acetylsalicylsäure auch im venösen System wirkt, ist bisher nicht gut untersucht.

Patienten und Methodik: In die ASPIRE-Studie wurden 822 Patienten mit venösen Thromboembolien oder Lungenembolien eingeschlossen nachdem die initiale Behandlung mit Antikoagulanzien abgeschlossen war. Dann wurden die Patienten entweder auf $100 \mathrm{mg}$ Acetylsalicylsäure oder Placebo randomisiert und für vier Jahre nachbeobachtet. Der primäre Endpunkt waren erneute venöse Thromboembolien und der sekundäre Endpunkt die Häufigkeit venöser Thromboembolien, Myokardinfarkte, Schlaganfälle oder kardiovaskuläre Todesfälle. Die Patienten waren im Mittel 55 Jahre alt und hatten überwiegend tiefe Beinvenenthrombosen. Bei den meisten Patienten war eine Antikoagulation über einen Zeitraum von sechs bis zwölf Monaten durchgeführt worden. Diese bestand bei dem Löwenanteil der Patienten aus niedermolekularem Heparin in der Akutphase und Warfarin in der Langzeittherapie.

Ergebnisse: Nach einer medianen Beobachtungszeit von 37,2 Monaten kam es bei 73 von 411 Patienten in der Placebogruppe und 57 von 411 Patienten in der Acetylsalicylsäuregruppe $\mathrm{zu}$ einem erneuten venösen thromboembolischen Ereignis ( Abb. 1). Dies entspricht einer Risikoreduktion zugunsten von Acetylsalicylsäure von $26 \%$ ( $p=0,09$, nicht signifikant). Acetylsalicylsäure führte allerdings zu einer signifikanten Reduktion von zwei vordefinierten sekundären Endpunkten: zu einer $34 \%$ igen Reduktion von venösen Thromboembolien, Myokardinfarkt, Schlaganfall oder kardiovaskulären Todesfällen und einer 33\%igen Reduktion von venösen Thromboembolien, Myokardinfarkt, Schlaganfall, Tod oder schweren Blutungskomplikationen. Schwerwiegende Blutungskomplikationen waren zwischen Placebo und Acetylsalicylsäure nicht unterschiedlich.

Schlussfolgerungen: Niedrig dosierte Acetylsalicylsäure führt nach einer Antikoagulationsbehandlung bei Patienten mit

Brighton TA, Eikelboom JW, Mann K et al; ASPIRE Investigators. Low-dose aspirin for preventing recurrent venous thromboembolism. N Engl J Med 2012; 367: $2039-41$ venösen Thromboembolien nicht zu einer Reduktion von erneuten venösen Thromboembolien. Die Behandlung reduziert allerdings die Kombination von venösen Thromboembolien und anderen vaskulären Endpunkten.

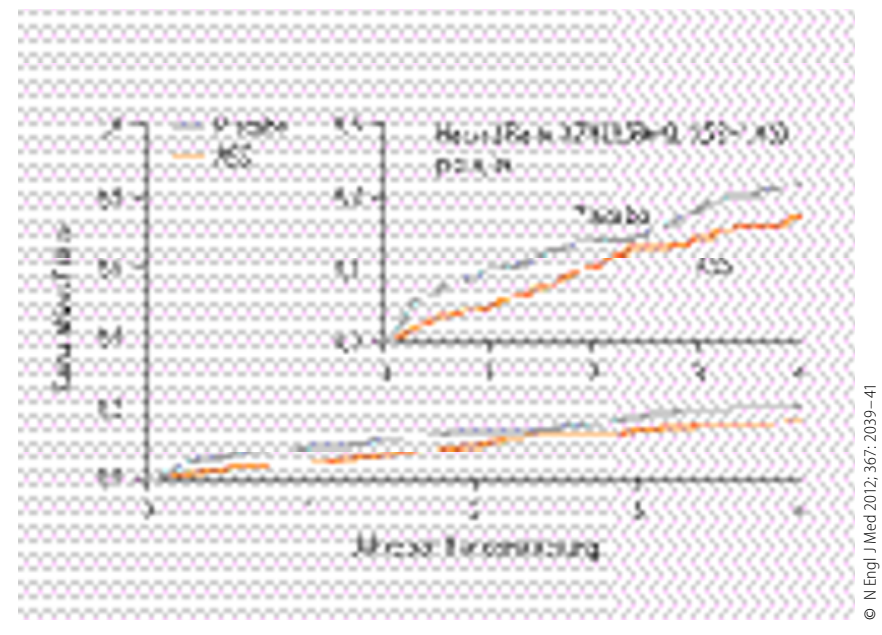

1 Kumulatives Risiko einer erneuten venösen Thromboembolie.

\section{-Kommentar von Hans-Christoph Diener, Essen}

\section{Das Lehrbuchwissen ändert sich}

Die ASPIRE-Studie ändert ganz offensichtlich Lehrbuchwissen, denn die Studie ergab einen starken Trend, dass Acetylsalicylsäure auch erneute venöse Thromboembolien verhindern kann. Die Autoren führten eine vorgeplante Metaanalyse mit einer zweiten Studie durch, die Aspirin im Vergleich zu Warfarin bei Patienten mit venösen Thromboembolien untersuchte, die WARFASA-Studie. Nimmt man beide Studien zusammen, kommt es zu einer $32 \%$ igen signifikanten Reduktion von venösen Thromboembolien, zu einer 34\%igen Reduktion von vaskulären Ereignissen und einer nicht signifikanten Zunahme von schwerwiegenden Blutungskomplikationen um 47\%. Das Ergebnis der beiden Studien ist wichtig, da sie darauf hinweisen, dass nach Ablauf einer oralen Antikoagulation nach tiefen Beinvenenthrombosen oder Lungenembolien eine Langzeitbehandlung mit Acetylsalicylsäure erfolgen sollte.

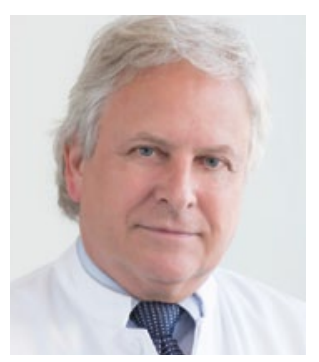

Prof. Dr. med. Hans-Christoph Diener, Essen

Direktor der Klinik für Neurologie, Universitätsklinikum Essen

E-Mail:h.diener@uni-essen.de 